$$
\text { CONF- } 960784--3
$$

TITLE: QUANTUM BREATHERS IN NONLINEAR PHONON AND COUPLED ELECTRON-PHONON SYSTEMS $\because=r+r=0$

SEP 0 ดी

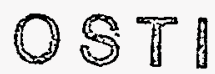

AUTHOR(S): J. Tinka Gammel, LANL.

Wen-Zheng Wang, Beijing, China, Chinese Academy of Sci. Alan R. Bishop; LANL

Markku I. Salkola, McMaster U., Ontairo Canada

SUBMITTED TO: Internationl Conf on Science \& Technology of Synthetic Metals (ICSM'96) Meeting

(Proceedings)

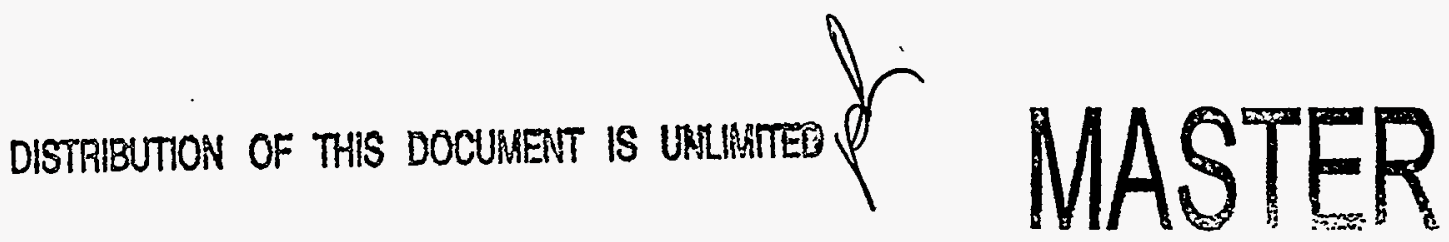

By acceptance of this article, the publisher recognizes that the U.S. Government retains a nonexclusive, royalty-free license to publish or reproduce the published form of this contribution. or to allow others to do so. for U.S. Government purposes.

The Los Alamos National Laboratory requests that the publisher identify this article as work performed under the auspices of the U.S. Department of Energy.
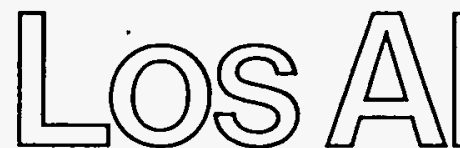


\section{DISCLAIMER}

Portions of this document may be illegible in electronic image products. Images are produced from the best available original document. 


\section{DISCLAIMER}

This report was prepared as an account of work sponsored by an agency of the United States Government. Neither the United States Government nor any agency thereof, nor any of their employees, makes any warranty, express or implied, or assumes any legal liability or responsibility for the accuracy, completeness, or usefulness of any information, apparatus, product, or process disclosed, or represents that its use would not infringe privately owned rights. Reference herein to any specific commercial product, process, or service by trade name, trademark, manufacturer, or otherwise does not necessarily constitute or imply its endorsement, recommendation, or favoring by the United States Government or any agency thereof. The views and opinions of authors expressed herein do not necessarily state or reflect those of the United States Government or any agency thereof. 


\title{
Quantum breathers in nonlinear phonon and coupled electron-phonon systems
}

\author{
J. Tinka Gammel ${ }^{a}$, W. Z. Wang ${ }^{a, b}$, A. R. Bishop ${ }^{a}$, and M. I. Salkola ${ }^{c}$ \\ a Theoretical Division, Los Alamos National Laboratory, Los Alamos, New Mexico 87545, USA \\ ${ }^{b}$ Institute for Theoretical Physics, Chinese Academy of Science, Beijing 100080, China \\ c Department of Physics \& Astronomy, McMaster University, Hamilton, Ontario L8S 4M1, Canada
}

\begin{abstract}
Nonlinear quantum phonon excitations are studied in 1D lattice models for both effective nonlinear phonon and coupled electron-phonon systems. Multi-phonon bound states exist which are eigenstates of the system Hamiltonian, and which are natural counterparts of breather solutions of classical nonlinear systems. These quantum breather states form particle-like bands and are characterized by a finite coherence length. Due to their structure and dynamics, quantum breathers have specific experimentally observable responses in many correlation functions. In the pure phonon model, the dynamic structure factor is found to have significant intensity for the breather states, with a corresponding quenching of the neighboring bands of multi-phonon extended states. In the coupled electron-phonon model, IR and optical absorption spectra for breathers are reported.
\end{abstract}

Keywords: models of nonlinear phenomena, lattice dynamics, computer simulations

The paradigms of nonlinearity have provided numerous insights into condensed matter physics [1]. For example, the dynamics of nonlinear excitations (such as solitons, polarons, and breathers) are central to understanding thermodynamic and transport properties of various low dimensional materials [2]. As an important example of intrinsic dynamic nonlinearity, breather excitations spatially localized and time-periodic waves in the form of bound states of linear excitations - have been found or excluded in various nonlinear models depending on the properties of nonintegrability and discreteness [3]. In particular, although breathers are rigorously stable in integrable partial differential equations, they are unstable in nonintegrable continuous systems. Recently it has been appreciated [4] that they can be stablized by lattice discreteness and strong nonlinearity. We are left with a central question for physical problems described by discrete, nonintegrable quantum systems: do nonlinear solutions analogous to the classical breather exist and, if so, what are their observable signatures?

To demonstrate the existence of quantum breathers we examined two simple Hamiltonians: (1) a pure-phonon single well $\phi^{4}$ model and (2) a coupled electron-phonon Holstein-Hubbard model. We study this problem by numerical exact diagonalization using an Einstein basis for the phonons. The only numerical approximation is the truncation of the infinite phonon Hilbert space. The studies of the $\phi^{4}$ model were reported in Ref. [5], and we refer the reader to this paper for the details of the Hamiltonian and method.

In Ref. [5], we examined in detail the low-lying eigenstates of the $\phi^{4}$ model. In contrast to a linear lattice, the ground state is isolated, with a finite gap to the excited eigenstates. The first band above the ground state is broad and mainly formed from extended one phonon states. There are several different kinds of higher excited bands of states, including isolated bound phonon bands with small but finite bandwidths which demonstrate particle-like coherence characteristic of breathers. The broader "continuum" bands by contrast show a significantly longer spatial correlation lengths. The distinctive local structure and dynamics of the breathers forming these narrow bands can be probed by neutron scattering [6], which directly measures the dynamic structure factor $S(q, \omega)$. In the linear case, one would see a response in $S(q, \omega)$ which traces each multi-phonon band dispersion, falling off exponentially in $q$ (quantum DebyeWaller factor) and decreasing algebraically with $\omega$, with regular spacing in $\omega$. Our prediction for the $\phi^{4}$ model is shown in Fig. 1. The elastic response of the ground state at $\omega=0$ has now gained an exponentially decaying $q$-dependence which is different from the harmonic Debye-Waller factor [6]. We also note that in this zero temperature calculation there is no zero-frequency contribution from the breathers [7]. In addition to the expected large low-energy contributions from the ground state and 


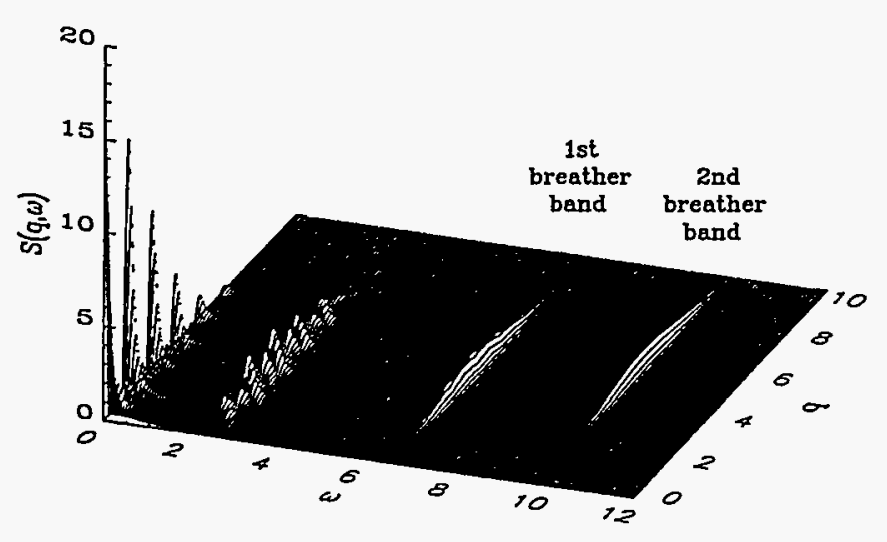

Fig. 1. Dynamic structure factor $S(q, \omega)$ of the quantum phonon $\phi^{4}$ model at zero temperature. Note that the equal-height $\delta$-peaks of $S(q, \omega)$ at $q=0$ are not shown.

the first phonon band, breather excitations are the dominant contribution, whereas those contributions from the extended multi-phonon "continua" are almost negligible. The quenching of the extended-state contribution is consistent with breathers forming as bound states of nearby phonons, and is similar to the transfer mechanism of the optical oscillator strength to local modes in the presence of electronic bound states. The center of the response is shifted to higher $q$ for higher breather bands, consistent with the fact that higher lying breathers (binding more phonons) are narrower in the classical limit. Another clear feature of this nonlinear system revealed by $S(q, \omega)$ is that the breather bands are irregularly spaced.

In the coupled electron-phonon Hubbard-Holstein model the low-lying eigenspectrum is fairly well described by phonon excitations in the electronic ground state. Analogous to the pure phonon single well $\phi^{4}$ model just discussed, bound phonon bands (breathers in the electronic ground state) are found with signatures very similar to the pure phonon model. However, the phonon dressing of the higher lying electronic states is also of keen interest. To probe the phonon excitation spectrum near the exciton at approximately $U-V$ and electronic band gap at approximately $U$, we have calculated the first order optical susceptibility, shown in Fig. 2. Interestingly, the response near the exciton shows only a single phonon peak. We are investigating whether this may be an "exciton breather".

In summary, we have identified quantum breathers as robust nonlinear solutions with their own characteristic spatial localization and multi-frequency dynamics. We predict that the dynamic structure factor is strongly enhanced at momentum and energy transfers corresponding to breather excitations and correspondingly reduced elsewhere. The breather is further identified in coupled electron-phonon systems by its optical signature. This result should apply in more general situations and provide exciting new possibilities for the identification of breathers and other nonlinear excitations. The numerical approach used here is readily applied to other nonlinear and electron-phonon coupled models.

We thank D. Cai, S.R. White, J. Zang, and and E.Y. Loh, Jr. for helpful discussions. This work was performed at the Advanced Computing Laboratory of LANL under the auspices of the US DOE. MIS was also supported in part by Natural Sciences and Engineering Research Council of Canada, and the Ontario Center for Materials Research.

[1] A.R. Bishop, J.A. Krumhansl and S.E. Trullinger, Physica D 1, 1 (1980).

[2] Nonlinearity in Condensed Matter, eds. A.R. Bishop, et al., (Springer-Verlag, 1987).

[3] M. Peyrard and M.D. Kruskal, Physica D 14, 88 (1983); D.K. Campbell and M. Peyrard, in Chaos, ed. D.K. Campbell, (American Institute of Physics, N.Y., 1980), pp. 305; S.R. Phillpot, A.R. Bishop and B. Horovitz, Phys. Rev. B 40, 1839 (1989).

[4] D. Cai, A.R. Bishop and N. Grønbech-Jensen, Phys. Rev. E 52, R5784 (1995); S. Takeno, in Nonlinear Coherent Structures in Physics and Biology, eds. K.H. Spatschek and F.G. Mertens (Plenum, 1994), pp. 39; S. Flach and C.R. Willis, ibid., pp. $59 ; 63$.

[5] W.Z. Wang, J.T. Gammel, A.R. Bishop, and M.I. Salkola, Phys. Rev. Lett. 76, 3598 (1996).

[6] See, e.g., M.I. Salkola, A.R. Bishop, S.A. Trugman, and J. Mustre de Leon, Phys. Rev. B 51, 8878 (1995).

[7] W.C. Kerr, D. Baeriswyl and A.R. Bishop, Phys. Rev. B 24, 6566 (1981).

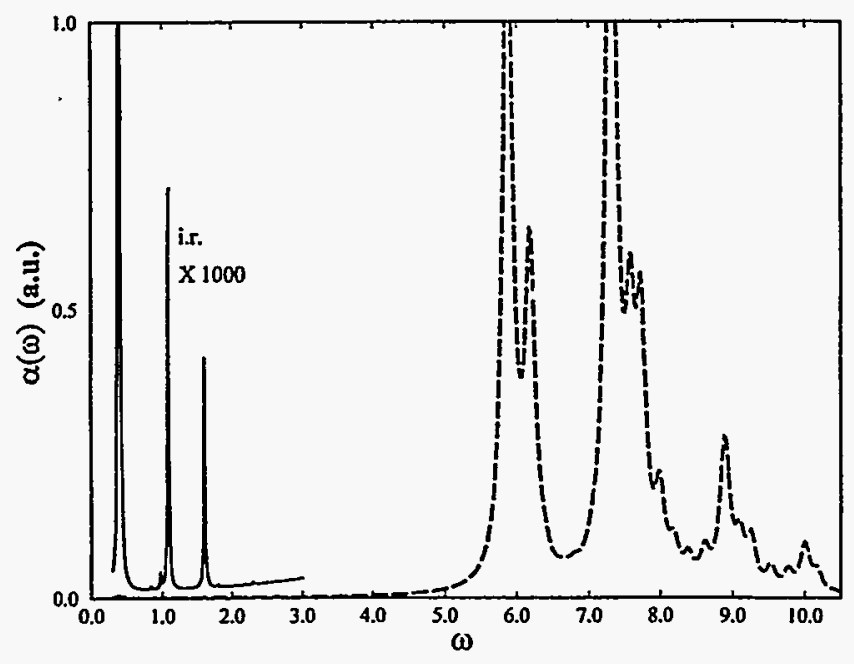

Fig. 2. First order optical susceptibility of the Holstein-Hubbard model with $U=8.1, V=1.11$. Note the sideband near the exciton at $U-V$ shows only a single phonon peak, in contrast to the electronic band at $U$ which shows multiple phonon bands. 